\title{
ENHANCEMENT OF ORAL UPTAKE OF AMIKACIN USING COPOLYMERS
}

\author{
Mohammad Amir*, Vaseem Ahamad Ansari, Anup Kumar Sirbaiya, Mohammad Zishan \\ Faculty of Pharmacy, Integral University, Dasauli, Kursi Road, Lucknow, India-226026
}

\section{ABSTRACT}

Amikacinis a semisynthetic derivative of Kanamycin; it was approved for clinical use in the U.S. in 1976. Amikacin is broadspectrum and potent aminoglycoside with limited clinical use owing high dose requirement.Many gram-negative bacteria, including many strains of Pseudomonas, Enterobacter, and serratiaare inhibited by $1-20 \mathrm{mcg} / \mathrm{ml}$ amikacin in vitro. After injection of $500 \mathrm{mg}$ of amikacin every 12 hours $(15 \mathrm{mg} / \mathrm{kg} / \mathrm{d})$ intramuscularly, peak levels in serum are $10-30 \mathrm{mcg} / \mathrm{ml}$. Amikacinis valuable because it was more active to aminoglycoside inactivating bacterial enzymes than is gentamicin. Since it was more inflated, amikacin was reserved for treatment of infections with gentamicin-resistant organisms. Peak plasma concentration should be kept between 20-30 mg/ml and trough concentration below $10 \mathrm{mg} / \mathrm{ml}$. There is no oral form of Amikacinis available as it is not absorbed orally. Various research on oral formulation of amikacinare going onsuch assignificantly improved oral uptake of amikacin in fvb mice in the presence of crl1605 copolymer, liposomal amikacin dry powder inhaler effect of fines on in vitro performance, thiolated chitosan nanoparticles as an oral delivery system for amikacin.

Key Word: Gram-negative bacteria, aminoglycoside,chitosan nanoparticles.

Article Info: Received 24 April, 2017; Review Completed 08 May, 2017; Accepted 08 May, 2017; Available online May 15, 2017

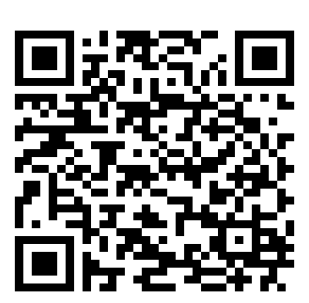

Cite this article as:

Amir M, Ansari VA, Sirbaiya AK, Zishan M, Enhancement of oral uptake of amikacin using copolymers, Journal of Drug Delivery and Therapeutics. 2017; 7(3):44-49

DOI: http://dx.doi.org/10.22270/jddt.v7i3.1449

*Address for Correspondence

Mohammad Amir, Research Scholar, Faculty of pharmacy, Integral University, Dasuli, Kursi, Road, Lucknow, 226026 E-mail: mdamir2436@gmail.com, Mob. No.+91-9559502908

\section{INTRODUCTION}

\subsection{Improvement of oral uptake of amikacin in mice}

This research was going oral uptake of amikacin improved by crl-1605 copolymer because amikacin is a highly polar molecule and is insoluble in lipid at physiological $\mathrm{pH}$ it exists as polycations. Amikacin drugs can't be given orally because it cannot absorb from the intestine. However intramuscular injection of amikacin rapidly absorbed and its peak plasma concentration is observed usually immediately after 30 min. and intravenous administration produces peak plasma concentration after infusion. The common phenotype is mediated by the presence of a membrane located pump, P-glycoprotein which facilitates the energy dependent transport of drugs out of the cell ${ }^{\mathbf{1 , 2}}$. The P-glycoprotein is related to a family of transport proteins, ATP-binding cassette transporters ${ }^{\mathbf{3 , 4}}$. Oral absorption of some drugs is also inhibited by $\mathrm{P}$ glycoprotein pumps on brush border of intestinal mucosa. Therefore, P-glycoprotein inhibitors may have potential to transport drug through gut, which is otherwise poorly absorbed after oral administration. Nonionic block copolymers synthesized from ethylene oxide and propylene oxide were developed specifically for use as surfactants. Because the sizes and relative positions of hydrophilic poly-oxy-ethylene and polyoxy-propylene blocks can be altered during synthesis, copolymers with significantly different surfactant 
characteristics can be produced. The CRL polymer containing 9000-20,000 Daltons poly-oxy-propylene (POP) cores flanked by poly-oxy-ethylene (POE) blocks that make up $2.52 \%$ of the total molecular weight. The high molecular weight CRL copolymer like (XL-1605 is soluble in aqueous buffer at concentrations up to 70 $\mathrm{mg} / \mathrm{ml}$ at temperature below $8^{0} \mathrm{C}$. The temperature increases through the cloud point, the copolymer molecules aggregate into uniformly sized but complex detergent micro-particles or micelles in which the hydrophobic POP portion of each molecule is positioned internally and the hydrophilic POE components exposed to the external aqueous environment. Because (XL-1605 can inhibit P-glycoprotein pump, It was observed the possibility of delivering amikacin orally in mice using CRL 1605 copolymer as a vehicle. To Chinnaswamy Jagannath knowledge, this was the first report of significantly improved uptake of amikacin when delivered orally in an animal or human model.

\subsection{Liposomal inhaler of amikacin: effect of fines on in vitro performance}

Amikacin sulfate is a broad-spectrum and potent aminoglycoside with limited clinical use owing to a high dose requirement and renal and audio-vestibular apparatus toxicity ${ }^{\mathbf{5}, \mathbf{6}}$. The major drawbacks associated with the use of earlier or conventional liposomal formulations are the tendency of liposomes to leak drug while in circulation, the extensive uptake of these liposomes by tissues of reticula endothelial systems (RES), and the inability of liposomes to extravagate into infected tissue ${ }^{7,8}$. Therefore, localized liposomal amikacin delivery was considered for the treatment of cystic fibrosis infections in the lungs. Liposomal encapsulation of Amikacin will give the required release of drug for longer time duration at the localized site, thereby reducing both the chances of systemic side effects and the frequency of dosing ${ }^{9}$

\subsection{In vitro and $e x$ vivo evaluations of amikacin oral delivery system}

The pharmaceutical research focus was being steadily shifted from the development of new chemical entities to the development of novel drug delivery systems (NDDS) of existing drug molecules to maximize their effectiveness in terms of therapeutic action. Effective slow-release and permeation enhancing regimens have demonstrated superior patient compliance ${ }^{\mathbf{1 0}}$.Thiolated polymers (Thiomers) are mucoadhesive polymers which displaythiol group bearing side chains. The backbone consists of safe polymers such as Chitosan that modify the release pattern of drugs. The interest is growing to develop mucoadhesive DDS that will attach to a tissue or to the surface coating of the tissue for the targeting of various absorptive mucosa. A biodegradable polymer such as Chitosan, a naturally polycationic biocompatible, biodegradable and non-toxic copolymer, was an established mucoadhesive and absorption enhancing polymer ${ }^{\mathbf{1 1}}$. Chitosan exhibits poor solubility at $\mathrm{pH}$ values above 6 that prevents enhancing effects at sites of absorption of drugs ${ }^{\mathbf{1 2}}$. Thiomers exhibit mucoadhesive, permeation enhancing, controlled release as well as enzyme and efflux pump inhibitory and tight junctions opening properties. These features make the
Thiomer technology highly interesting for non-invasive drug delivery routes. In the presence of $0.5 \%$ thiolated polycarbophil, for instance, the apparent permeability coefficient of insulin could be improved ${ }^{13}$. Thiomers can be directly given compressed to tablets or given as solutions ${ }^{14}$. The potential of applications of such mucoadhesive polymers for the delivery of small organic molecules, proteins, vaccines and DNA are already discus about it but still experiments regarding the delivery of antibiotics such as Amikacin using Thiomers use as drug delivery vehicles are lacking. Oral drug delivery to accomplish intracellular effects of such highmolecular weight hydrophilic agents needs to be coupled with the use of an absorption-enhancing agent, which is safe and biodegradable. Colloidal drug carriers such as liposomes and nanoparticles are easily taken up by phagocytic cells and accumulate in the organs of the reticula endothelial system (RES). Therefore, they hold promise as carriers for many different drugs including antibiotics used for the treatment of intracellular infections that would not normally find easy access into intracellular sites. Amikacin was a polycationic aminoglycoside. Aminoglycosides are water-soluble weak bases that were polycations at body $\mathrm{pH}$. Aminoglycosides cannot be delivered orally probably due to the efflux by P-glycoprotein pumps in the brush border of intestine and their high solubility in water ${ }^{\mathbf{1 5}}$. More recently Werle and Hoffer revealed efflux pum inhibitory capability of Thiomers ${ }^{16}$. The thiol groups on Thiomers can be, to some extent, oxidized under mild conditions to form intermolecular covalent bonds, and as a result, modulate water absorption and swelling behavior of nanoparticles to improve drug delivery and release ${ }^{17}$.It was trying to demonstrate that amikacin could be loaded in thiolated nanoparticles to increase its absorption from intestinal tract by increasing the mucoadhesion of the particles and facilitating nanoparticle uptake by cells. These particles later on could also be utilize as vehicles to deliver amikacin by tracheal routes against obligate aerobes such as Mycobacterium Tuberculosis.

\section{DISCUSSION}

\subsection{Improvement of oral uptake of amikacin in mice}

It was observed a good correlation between the target amikacin concentration and observed concentration of amikacin in normal mouse serum supplemented with known amount of amikacin. Therefore, the fluorescence polarization immunoassay for amikacin which is intended for monitoring concentrations of amikacin in human serum can also be used to measure the concentration of amikacin in sera of mice. Inexperiments take separate series to fed mice with CRL 1605 and collected blood after $1 \mathrm{~h}$ and $2 \mathrm{~h}$. Sera were separated from blood cells by centrifugation. Sera were supplemented with known concentration of amikacin. The target concentration of amikacinwas $8.0 \mu \mathrm{g} / \mathrm{ml}$. In the serum containing CRL -1605 and collected $1 \mathrm{~h}$ after gavage, the observed concentration of amikacin was $7.7 \mu \mathrm{g} / \mathrm{ml}$. Similarly, the serum collected after $2 \mathrm{~h}$ of gavage and then supplemented in vitro with amikacin, the observed concentration of Amikacin was $7.9 \mu \mathrm{g} / \mathrm{ml}$. Again, the target concentration of amikacin was 8.0 
$\mu \mathrm{g} / \mathrm{ml}$. Therefore, we conclude that CRL 1605 has no effect in the fluorescence polarization immunoassay of amikacin. In the first set of experiments to estimated serum concentration of amikacin at a 1 hour interval. In this experiment, it was not only demonstrated significantly elevated serum amikacin level in mice in the presence of CRL1605, but also demonstrated none detected amikaoin levels when vehicle control or CRL 1605 copolymer were used for gavage. In this experiment mice divided four group and used three mice in each group. This experiment also established that the higher concentration of amikacin in mice receiving amikacin along with the copolymer CRL 1605 was due to improved gastrointestinal absorption and not due to cross reactivity of CRL -1605 with the arnikacin antibody in the amikacin immune assay. In separate sets of experiments, there was used five mice in each groups. The first group was control where mice received amikacin alone. The second group of mice received amikacin along with the copolymer GRL 1605. It was observed significantly elevated concentrations of arnikacin in the second group of mice compared to the first group (controls), indicating that oral uptake of amikacin was increased significantly in the presence of CRL-1605, In experiments one through and five used relatively high dose of amikacin and observed significantly improved oral uptake of amikacin in the presence of copolymer CRL-1605. Interestingly when we used lower dose of amikacin $(100 \mathrm{mg} / \mathrm{Kg})$ in experiment 6 , and again observed significantly improved oral uptake of amikacin in the presence of CRL 1605. Therefore, accomplish that in both doses, CRL-1605 is effective in delivering amikacin orally in mice. These preliminary experiments indicate a potential for delivering amikacin orally in man using CRL-1600 copolymer as a vehicle. Other aminoglycosides may also be delivered using this approach because all aminoglycosides are polar and exist as polycations at physiological PH. Therefore, other aminoglycosides can also be incorporated into CIU-1605 vehicle. In assumption, it was demonstrated that the oral uptake of amikacin is significantly improved in the presence of copolymer CRL-1605. Because amikacin was a relatively inexpensive drug and CRL-1605 is nontoxic (unpublished data, available on request from cytRx Corporation), it was possible to use large dose of amikacin in oral formulation. However, mice and human may behave differently in the uptake of amikacin in the presence of the copolymer. Our goal is study structurallyrelated polymers such as CRL 1072 in order to investigate which polymer produces best oral uptake of amikacin concentrations in sera of mice in the presence and absence of CRL 1605 Amikacin will also consider the possibility of delivering other aminoglycosides, for exampletobramycin orally using CRL 1605 and other structurally related copolymers.

\subsection{Liposomal inhaler of amikacin: effect of fines on in vitro performance}

The liposomal amikacin were prepared using modified reverse phase evaporation (REV) technique using alternative organic solvents such as ethyl acetate and ethanol (1:1). The organic solvents such as methanol or diethyl ether employed in the liposome preparation (although usually removed by evaporation) may remain as traces in the final formulation and can lead to a possible risk for human health and inadequate stability of the vesicles ${ }^{18}$. Use of other organic solvents such as ethanol and ethyl acetate can solve this problem. Ethanol forms a monophasic system upon contact with aqueous phase, while ethyl acetate forms a biphasic system (emulsion) upon contact with aqueous phase. When ethyl acetate was used alone, it resulted in distorted spherical vesicles, which may be due to formation of an unstable biphasic system upon contact with the aqueous phase. Use of ethanol alone resulted in high percentage drug entrapment (PDE), which may be due to formation of a monophasic system upon contact with the aqueous phase. However, drug leakage was observed due to the presence of traces of ethanol leading to disruption of the bilayer. In the case of ethyl acetate: ethanol (1:1) combination, proper spherical vesicles and high PDE were observed. Combination of these organic solvents with aqueous phase forms a stable emulsion, which is a prerequisite for REV ${ }^{19}$. When the aqueous phase to organic phase ratio was raised from 1:3 to 1:5, marked increase in the PDE was observed. Further increase in the organic phase did not result in increase in PDE. The prepared liposomes were found to be multilamellar and were identified by the presence of Maltese crosses in liposomes. The prepared liposomes were extruded by passing through $2 \mu \mathrm{m}$ polycarbonate membranes to a reproducible mean liposomal size below $5 \mu \mathrm{m}{ }^{\mathbf{2 0}}$.The liposomal dispersion was dialyzed and free drug was removed. PDE in liposomes was $96.7 \pm 1.9$ and $98.5 \pm$ 1.4 for AMK1 and AMK2, respectively. Laser light scattering microscopy revealed mean liposomal sizes for AMK1 and for AMK2. Difference in liposomal size may be due to the presence of a different charge present on the liposomal surface. The prepared liposomes were lyophilized using appropriate optimized and cryoprotectant for the drug retained in lyophilized liposomal amikacin as described previously ${ }^{\mathbf{2 1}}$. Liposomes were best preserved in their structure with PDR using sucrose as a cryoprotectant in mass ratio of lipid:sucrose at 1:4. During the freeze-drying process of liposomes, liposomes constrict and get coated on the optimum surface of crystallized sugar. Hydration of polar head groups with the hydroxyl group of sucrose leads to stabilization of liposomes. If the concentration of sucrose was less than optimum, the crystallized sugar does not provide adequate surface for the adherence of the constricted bilayer leading to drug leakage. Hence, it may be concluded that the bulk concentration of sugar required as cryoprotectant depends on the type of sugar selected and saturation of the polar head groups of the bilayer by drug or other formulation components. The lamellarity and size of liposomes were expected to change these requirements. To formulate AMK LDPI formulation, a series of experiments was conducted. Lyophilized liposomes when formulated as an LDPI formulation without using any carrier molecule resulted in low FPF value. This observation describes the importance of the addition of lactose carrier in formulating the LDPI formulation. Pharmatose 325M was sieved to get 63 - to $90-\mu \mathrm{m}$ size range fraction as a carrier to formulate LDPI formulations. The lyophilized liposomes were mixed with sieved Pharmatose $325 \mathrm{M}$ in 
the range of liposome: lactose mass ratio from 1:1 to 1:6, and Pharmatose 325M's effect on FPF was studied The data revealed the optimum liposome: lactose mass ratio of 1:5. Optimum concentration of carrier is required to achieve detachment of liposomal drug from carrier molecule. Carrier concentration of less or more than optimum resulted in low FPF or no further increase in FPF. The effects of fines in 5\%, 10\%, and $15 \%$ proportion (wt/wt) and mixing sequence with carrier and sieved lyophilized liposomes keeping the final liposome: lactose mass ratio of 1:5 were evaluated. Data revealed an optimum concentration of $10 \% \mathrm{wt} / \mathrm{wt}$ fines (sieved Sorbolac 400) and mixing sequence of fines with carrier and then with sieved lyophilized liposomes. At the $10 \%$ level of fines, high-energy adhesion sites (HA) of lactose may bind strongly to the carrier and low-energy adhesion sites (LA) may allow the formation of more reversible bonds with liposomal drug. This action results in efficient detachment of liposomal drug from the carrier as observed with plain DPI formulations 22 Hence, $10 \%$ sieved Sorbolac 400 (wt/wt) added to AMK LDPI formulation.

The EI of AMK2 was found to be better than the AMK1, suggesting more effective liposomal drug deposition into lung. This finding may be due to turbo-electrification or charge generation in liposomal powder during dispersion via the Rotahaler. The lower ratio of EI/FPF is suggestive of efficient dispersion of AMK1 from the device, but unlike the control more proportion of the dispersed powder has been deposited in the upper respiratory tract ${ }^{\mathbf{2 4}}$. Evaluation and control of flow and dispersion or deaggregation characteristics of the formulation are of critical importance in the development of DPI products. Interparticle forces that influence flow and dispersion properties are particularly dominant in micronized or microcrystalline powders required for inhalation therapy ${ }^{\mathbf{2 5 , 2 6}}$. It has been demonstrated that powder adhesion, mediated in part by Van der Waal forces, is directly related to particles ${ }^{27}$.

\subsection{In vitro and $e x$ vivo evaluations of amikacin oral delivery system}

\subsubsection{Synthesis of chitosan conjugates}

LMW Chitosan (15 KDa) soluble at $\mathrm{pH} 7$ was prepared. LMW Chitosan when formulated yields smaller and more uniform particles ${ }^{28}$ The carboxylic acid moieties of NAC and NAP were activated applying EDAC [1ethyl-3-(3-

dimethylaminopropyl)carbodiimide].Chitosan-Cysteine conjugate was synthesized the same way as a control to compare with new thiomers (NAC, NAP conjugates). Chitosan was also prepared as control exactly following the same procedure as described but omitting EDAC during the coupling reaction. The lyophilized NAC Chitosan and NAP Chitosan appeared as odorless, white powders of fibrous structure. They easily swell in $\mathrm{pH}$ values below 7 and forma transparent gel of high viscosity. The lyophilized conjugates should be stored in cool temperature at $4^{0} \mathrm{C}$.FTIR analyses were conducted to demonstrate the success of the synthesis process.

\subsubsection{Determination of the thiol group content}

The thiol group content of the polymers was determined using iodometric method. The highest coupling rate was achieved at the polymer to NAP or NAC ratio of $1: 1$. Results showed that the amount of covalently bound groups could not increase by raising the share of NAP or NAC during the coupling reaction. When increasing the amount of EDAC added to activate the carboxylic moieties of the polymer augmented the amount of thiol groups immobilized on the polymer chain (Fig.1)

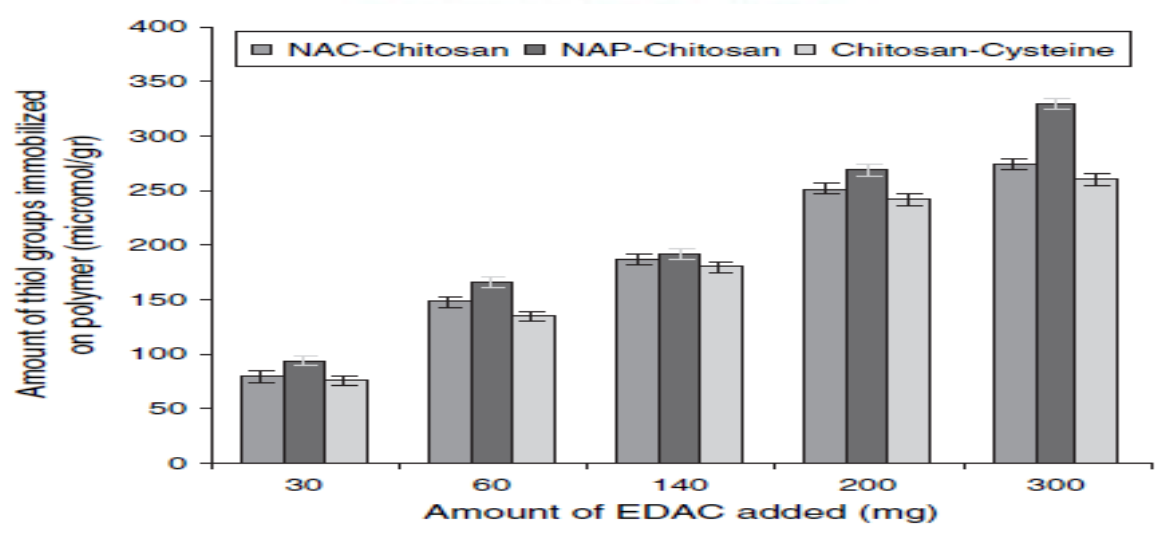

Figure 1: The influence of the amount of edac added on the amount of thiol groups

Another parameter affecting the thiol groups conjugation to Chitosan was the $\mathrm{pH}$ value of the reaction medium. Figure 4 shows results from conjugation of polymers in different $\mathrm{pH}$ values. It appears that setting the $\mathrm{pH}$ of the medium at 5 leads to higher amounts of thiol groups on the polymer in comparison to thiol groups obtained at lower or higher $\mathrm{pH}$ values. A pH value of 3.5 gives lower amounts of covalently attached thiol groups. At this pH-value, EDAC could not gain its full reactive potential. At a $\mathrm{pH}$-value of 5 the yield of polymer-bound of thiol groups is highest, as EDAC has gained full potential, but as the $\mathrm{pH}$-value increases, oxidation of sulfhydryl groups during the coupling reaction was too increase, producing fewer polymer bound thiol groups. 


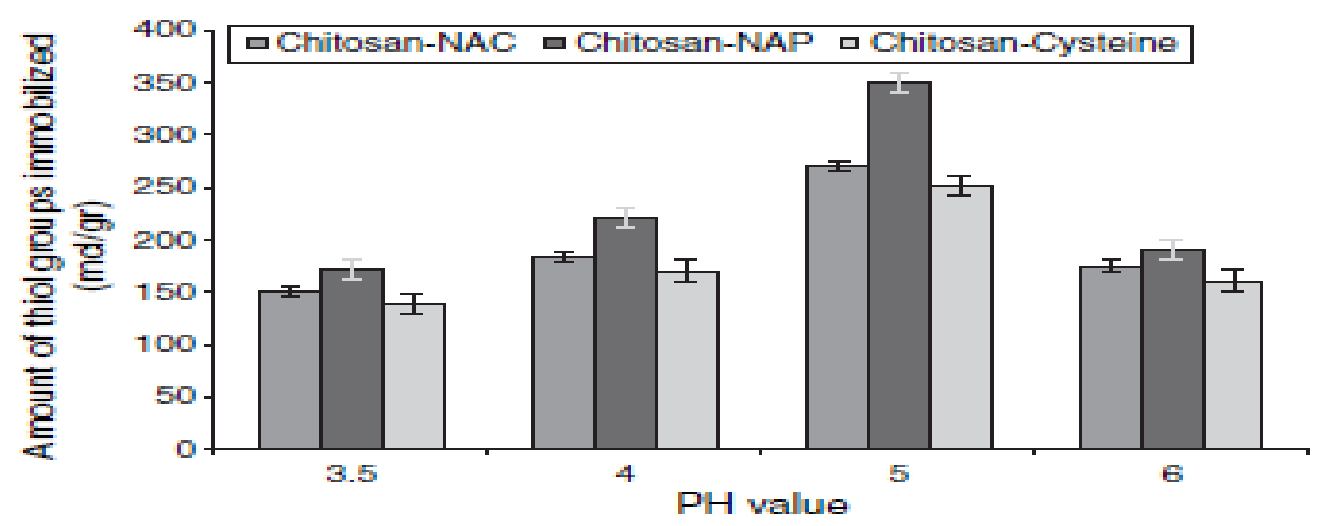

Figure 2: The influence of ph value on the amount of thiol groups immobilized on polymer

2.3.3. Disintegration studies-Results achieved of disintegration studies show that Thiomer tablets (polymers synthesized in a $\mathrm{pH}$ value of 5) were more soluble in acetate buffer compared to Chitosan tablets. This observation could be attributed to the presence of thiol and acetyl groups on the polymers and the use of LMW Chitosan which all contribute to higher polymer solubility in water.

\subsubsection{Drug permeation studies}

The results in vitro permeation studies were carried out using rat gut. The viability of the tissue was determined measuring the concentration of glucose.As glucose is actively transported, inside concentration the sac was higher than in the incubation solution and tissue viability could be maintained for up to $150 \mathrm{~min}$. shows that an acceptable amount of permeation in over $2 \mathrm{hrs}$ was achieved. According to the results $82 \%$ of the entrapped amikacin permeated through the rat gut membrane.

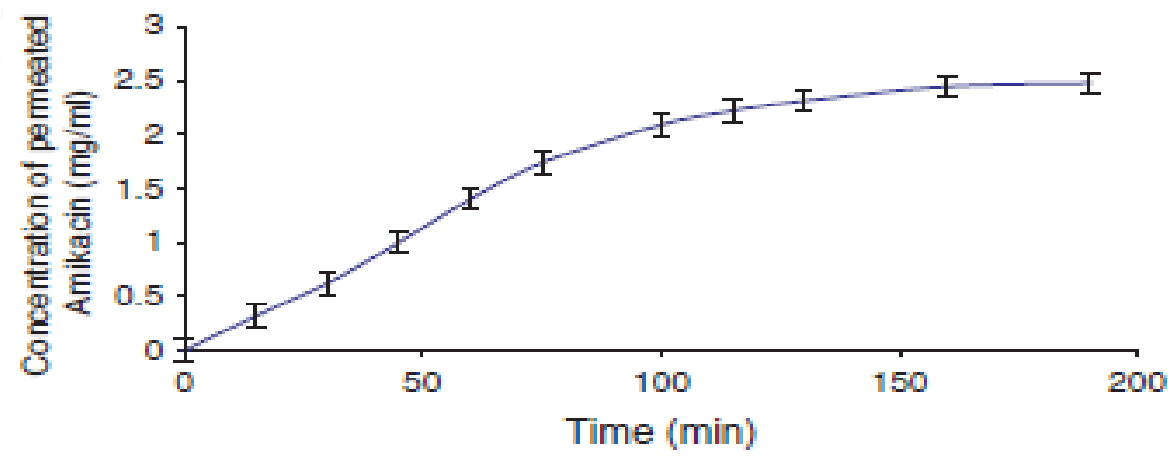

Figure 3: Time versus concentration of amikacin released from 2grof nap-chitosan-amikacin nanoparticles

\subsubsection{Tensile studies}

The results achieved by tensile studies demonstrate a 6 fold increase in mucoadhesion of NAC-Chitosan and a 7 fold increase in mucoadhesion of NAP-Chitosan compared to Chitosan control tablets. According to the results above experiments achieved NAP-Chitosan shows our desired properties and is the chosen polymer for future experiments.

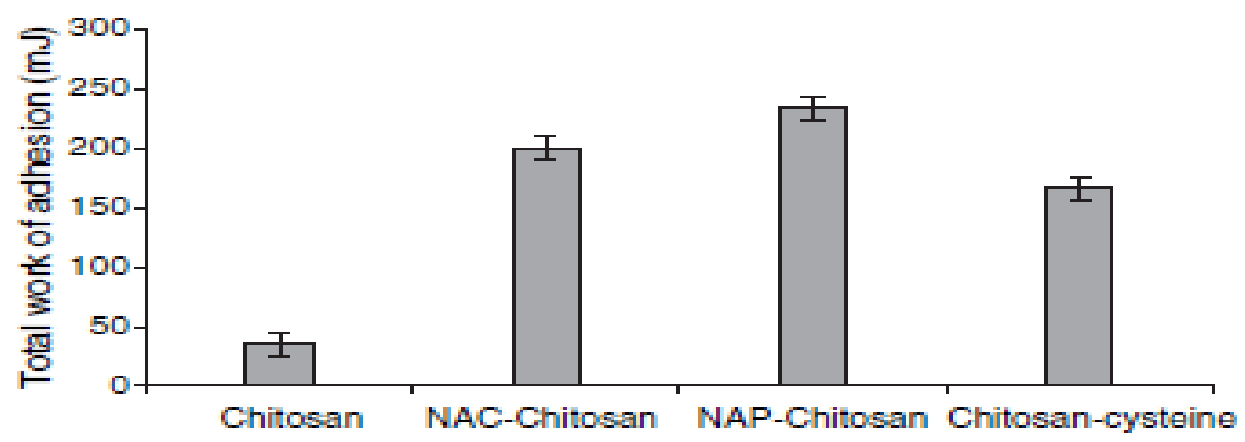

Figure 4: Twa results for thiomer and chitosan tablets 


\section{CONCLUSION}

Amikacinis a semisynthetic derivative of Kanamycin; it was approved for clinical use in the U.S. in 1976. Amikacin is broad-spectrum and potent aminoglycoside with limited clinical use owing high dose

\section{REFERENCES}

1. Preet MC, Lgor BR, Expression and activity of Pglycoprotein, a multidrug efflux pump, in human hematopoietic stem cells, Cell press, 1991; 66(1):85-94.

2. Fath MJ, and Kolter R, Microbiology and Molecular Biology, American society for microbiology, 1993; 57(4):995-1017.

3. Ouellete MD, Legare D., Papadopoulou B., Microbial multidrug-resistance ABC transporters, Trends Microbial, 1994; 2(10):407-411.

4. Kaidoh K, Kimura M, Miyauchi S, Nara T, Kamo N, Drug Extrusion in Corynebacterium Glutamicum, Microbial drug resistance, 2009; 3(4):345-350.

5. Kumana CR, Yuen KY, Parenteral aminoglycoside therapy: Selection, administration and monitoring, Adis International Ltd, 1994; 47(6):902-913.

6. Molitoris BA, Bruce A, Cell biology of aminoglycoside nephrotoxicity: newer aspects, Current Opinion in Nephrology \& Hypertension, 1997; 6:384-388.

7. Senior J, Fate and behavior of liposomes in vivo, Crit Rev Ther Drug Carrier Syst, 1987; 3(2):123-193.

8. Patel HM, Serum opsonins and liposomes: their interaction and opsonophagocytosis, The Drug Carrier Syst. 1992; 9(1):39-90.

9. Ganderton D, The generation of respirable cloud from coarse powder aggregates, Journal of Biopharm Sciences, 1992; 3:101-105.

10. Atyabi F, Manoochehri S, Moghaddam HS, and Dinarv R, Cross-linked starch microspheres: Effect of cross-linking condition on the microsphere characteristics, Arch. Pharm. Res, 2006; 29(12):1179-1186.

11. Jaiswal PK, Kesharwani S, Kesharwani R, Patel DK, Chitosan: a review on its varied novel therapeutic and industrial applications, Journal of Drug Delivery and Therapeutics. 2016; 6(6):70-79

12. Avadi MR, Jalali A, Mir A, Mohammad S, Shamimi K, Bayati KH, Nahid E, Dehpour AR, and MRafiee-T ehrani, Diethyl methyl chitosan as an intestinal paracellular enhancer: ex vivo and in vivo studies, Journal of Pharmaeutical, 2008; 293(1-2):83-89.

13. Clausen $\mathrm{AE}$ and Bernk $\mathrm{OA}$, In vitro evaluation of the permeation-enhancing effect of thiolated polycarbophil, Journal of Pharmaceutical Science, 2000; 89(10):1253-1261.

14. Roldo $M$, Hornof $M$, Caliceti $P$, and Bernk OA, Mucoadhesive thiolated chitosans as platforms for oral controlled drug delivery: synthesis and in vitro evaluation, Eur. J. Biopharmaceutics, 2000; 57(1):115-121.

15. Jagannath C, Wells A, Mshilvdadze M, Olsen M, Emanule RM, Hunter RL and Dasgupta A, Thiolated Chitosan Nanoparticles as an Oral Delivery System for Amikacin: In
requirement.Many gram-negative bacteria, including many strains of Pseudomonas, Enterobacter, and serratia are inhibited by $1-20 \mathrm{mcg} / \mathrm{ml}$ amikacin in vitro. in this article discussed about the oral uptake of Amikacin By three different formulation.inhance the oral uptake.

Vitro and Ex Vivo Evaluations, Lifesciences 2002; 64(9): 1773.

16. Werle $\mathrm{M}$ and Hoffer M, Glutathione and thiolated chitosan inhibit multidrug resistance $\mathrm{P}$-glycoprotein activity in excised small intestine, Journal of Controlled Release, 2006; 111(12):41-46.

17. Majzoob S, Atyabi F, Dorkoosh F, kafedjiiski k, Pectincysteine conjugate: synthesis and in-vitro evaluation of its potential for drug delivery, J Pharmacy and Pharmacology, 2006; 58(12):1601-1610.

18. Cortesi R, Esposito E, Gambarin S, Telloli P, Menegatti E, Nastruzzi C, Preparation of liposomes by reverse-phase evaporation using alternative organic solvent, J Microencapsul, 1999; 16(2):251-256.

19. Betageri GV, Jenkins SA, Parsons DL, Liposome Drug Delivery Systems, Lancaster, PA: Technomic Publishing Company, 1993; 52:16-17.

20. Staniforth JN, Preformulation aspects of dry powder aerosols, IL: Interpharm Press, 1996; 66(33):65-73.

21. Fiegel J, Fu J, Hanes J, Poly (ether-anhydride) dry powder aerosols for sustained drug delivery in the lungs, Journal of Control Release, 2004; 96:411-423.

22. Staniforth JN, Preformulation aspects of dry powder aerosols, Respiratory Drug Delivery V. Buffalo Grove, IL: Interpharm Press; 1996; 9(2):65-73.

23. Shah P, and Misra A, Liposomal Amikacin Dry Powder Inhaler: Effect of Fines on In Vitro Performance AAPS, Pharm Sci. Tech, 2004; 59(10):812-813.

24. Hino T, Serigano T, Yamamoto H, Takeuchi H, Niwa T, Kawashima Y, Particle design of wogon extract dry powder for inhalation aerosols with granulation method, International Journal of Pharmaeutical, 1998; 168(1):59-68.

25. Gonda I, Physico-chemical principles in aerosol-delivery, Germany: Medipharm Scientific Publishers, 1992; 9(12):95115.

26. Hickey AJ, Inhalation Aerosols - Physical and Biological Basis for Therapy, New York, NY: Marcel Dekker Inc, 1996; 11(9):441-473.

27. Hamaker HC, London-van der Waals forces attraction between spherical particles, Physica (Utrecht), 1937; 4(10):1058-1072.

28. Bernk OA, and Hornof $M$, and Margit M, Thiolated chitosans: development and in vitro evaluation of a mucoadhesive, permeation enhancing oral drug delivery system, Journal of Drug Delivery, 2001; 1(3):241-248.

29. Atyabi F, Talaie F, and Dinarvand R, Thiolated Chitosan Nanoparticles as an Oral Delivery System for Amikacin: In Vitro and Ex Vivo Evaluations, Journal of Nanoscience and Nanotechnology, 2009; 9(11):459-4603. 